\title{
Extraction and evolution of bubbles attributes in a two- phase direct contact evaporator
}

DOI:

10.1016/j.ijheatmasstransfer.2018.04.002

\section{Document Version}

Accepted author manuscript

Link to publication record in Manchester Research Explorer

\section{Citation for published version (APA):}

Xiao, Q., Gao, Q., Zhang, J., Xu, J., Pan, J., \& Wang, H. (2018). Extraction and evolution of bubbles attributes in a two-phase direct contact evaporator. International Journal of Heat and Mass Transfer, 124, 761-768.

https://doi.org/10.1016/j.ijheatmasstransfer.2018.04.002

\section{Published in:}

International Journal of Heat and Mass Transfer

\section{Citing this paper}

Please note that where the full-text provided on Manchester Research Explorer is the Author Accepted Manuscript or Proof version this may differ from the final Published version. If citing, it is advised that you check and use the publisher's definitive version.

\section{General rights}

Copyright and moral rights for the publications made accessible in the Research Explorer are retained by the authors and/or other copyright owners and it is a condition of accessing publications that users recognise and abide by the legal requirements associated with these rights.

\section{Takedown policy}

If you believe that this document breaches copyright please refer to the University of Manchester's Takedown Procedures [http://man.ac.uk/04Y6Bo] or contact uml.scholarlycommunications@manchester.ac.uk providing relevant details, so we can investigate your claim.

\section{OPEN ACCESS}




\title{
Extraction and evolution of bubbles attributes in a two-phase direct contact evaporator
}

\author{
Qingtai Xiao ${ }^{\mathrm{a}, \mathrm{b}}$, Qin $\mathrm{Gao}^{\mathrm{c}}$, Jing Zhang ${ }^{\mathrm{d}}$, Jianxin $\mathrm{Xu}^{\mathrm{a}, \mathrm{d}, *}$, Jianxin Pan ${ }^{\mathrm{e}, *}$, Hua Wanga,f,* \\ ${ }^{a}$ State Key Laboratory of Complex Nonferrous Metal Resources Clean Utilization, Kunming University \\ of Science and Technology, Kunming 650093, PR China \\ ${ }^{b}$ School of Mathematical and Statistical Sciences, The University of Texas Rio Grande Valley, Edinburg \\ Texas 78541, USA \\ ${ }^{c}$ Faculty of Science, Kunming University of Science and Technology, Kunming 650500, PR China \\ ${ }^{d}$ Quality Development Institute, Kunming University of Science and Technology, Kunming 650093, PR \\ China \\ ${ }^{e}$ School of Mathematics, University of Manchester, Manchester M13 9PL, UK \\ ${ }^{f}$ Faulty of Metallurgical and Energy Engineering, Kunming University of Science and Technology,
} Kunming 650093, PR China

\begin{abstract}
Understanding the bubble regimes is a fundamental step toward conducting heat transfer enhancement. The non-invasive measurement of mixing inside a direct-contact heat transfer process, using a direct video imaging technology, provides powerful opportunities for characterising the visual observations of the phenomena and quantifying the process complexities previously. Experimental bubble shape feature parameters were obtained by means of the photographic recording technique for a direct-contact evaporator. Four design factors with three levels respectively were analysed for the mixing system that involves the exchange of heat between two immiscible fluids (continuous and dispersed phases). Using the Ripley's $K$ function, new results are presented for two-phase flow mixing which can distinguish differences in the mixing behavior of dispersed phase. In all cases considered, quantitative comparisons of the evolution curves representing different experimental conditions were conducted with reported experimental data. Following the local mixing curve, the current results can also be processed to provide the mixing time found to be in good agreement with available data. The relationship between shape feature parameters of bubbles and volumetric heat transfer coefficient was found to be highly independent on experimental design parameters.
\end{abstract}

Keywords: direct-contact evaporation; dispersion and distribution; bubbles uniformity; shape feature parameters; image analysis.

\footnotetext{
${ }^{*}$ Corresponding authors at: State Key Laboratory of Complex Nonferrous Metal Resources Clean Utilization, Kunming University of Science and Technology, Kunming 650093, PR China (J. Xu, H. Wang) and School of Mathematics, University of Manchester, Manchester M13 9PL, UK (J. Pan).

Email addresses: qingtaixiao2016@kmust.edu.cn (Qingtai Xiao), xujianxina@163.com (Jianxin $\mathrm{Xu}$, jianxin.pan@manchester.ac.uk (Jianxin Pan), wanghua65@163.com (Hua Wang)
} 


\section{Introduction}

Although boiling is a complex process, it is a very efficient mode of heat transfer in various heat exchange systems [1]. Direct-contact boiling evaporators are widely employed as the chemical reactors in the industrial processes [2, 3]. The mixing quality and the 5 critical parameters (such as mixing uniformity of bubbles, droplets, or particles, local bubble size distribution, gas holdup, interfacial area, etc.) in reactor design and control have a great influence on the performance of contactors [4, 5]. Direct imaging technology is an effective and convenient method for the estimation of those critical parameters [6, 7]. To the best of our knowledge, many researchers have carried out this study thoroughly using numerous techniques including Doppler anemometry techniques [8], tomographic techniques [9], invasive probe techniques, and direct imaging techniques [10]. Additionally, image analysis with advanced mathematical methods, regarded as a normal practice, is gaining importance for object identification [11, 12].

On the one hand, mixing uniformity of objects (bubbles, droplets, or particles) has been considered [13, 14]. The purpose of mixing is to obtain homogeneous [15]. It has a decisive impact on the overall performance of reaction processes. There is therefore an increased desire for measuring and comparing mixing performance [16, 17]. The efficient evaluation of mixing uniformity is also required in the boiling heat transfer process which is one of the most efficient kinds of heat transfer processes widely used in numerous engineering systems. The resulting improvement heat transfer performance is believed on how uniformly the discrete phase is mixed into the continuous phase. The bubble detection problem is not easy to solve because the bubbles are not transparent when imaging inside a same industrial process, which causes the bubble appearance to vary. For granular materials, the nonuniformtiy of porosity distribution within a specimen was evaluated using imaging techniques [18]. The literature concerning bubbling is extensive [19]. There is also homogeneous bubbling regimes where the size of the bubbles present little variation in heat transfer process [3]. For the air-water system, a photographic methodology was used to experimentally determine in a direct-contact evaporator under different operating conditions [2]. Moreover, the authors also developed a sparger model for a non-coalescing system and using bubble formation models for isothermal and nonisothermal conditions [20]. Coënt et al. proposed a non-intrusive method to follow the blending of powders and viscous liquid in a classical reactor under different agitation conditions by taking a series of images and analyses them [21]. 
On the other hand, the shape feature parameters of bubbles are also considered. The bubble size distribution (BSD) of the discrete phases in multiphase systems such as the BSD of bubbles in gas-liquid or gas-liquid-solid systems is significant for the operation of the specific applications. The knowledge of BSD can enhance the understanding of mixing and heat and mass transfer properties for design and scale-up of the operations [10]. The direct imaging technique has been a common technique for BSD measurement particle image velocity techniques coupled with advanced image processing tools to measure local features of gas-liquid dispersions, such as bubble size, gas hold-up and interfacial area [24, 4, 5]. Kalbfleisch and Siddiqui (2017) experimentally investigated the influence of a mesh-type bubble breaker in a two-phase vertical co-flow using 45 high-speed imaging [25]. The effect of surfactant addition on void fraction distributions has been investigated by Kleinbart et al. [26]. Statistical theories coupled image processing techniques are receiving an increasing interest for measuring flow quantities and local bubble distribution in multiphase flow mechanically agitated vessels [27, 19]. It also provides powerful opportunities for characterising and quantifying the process complexities. Zafari et al. presented a method for segmentation of clustered partially overlapping objects with a shape that can be approximated using an ellipse [28].

Among those studies, several methods can be necessarily used to measure the critical parameters. Currently, a number of commonly used measurement techniques, able to access local information on dispersion properties has been devised over the years, including 55 phase Doppler anemometer [29, 30, 8, 31], capillary suction probe [29, 32], digital imaging analysis [29, 33, 34], etc. The local (probe) results are obviously not representative of the global state of mixing. Recently, statistical image analysis and feature extraction methods are used to quantitatively characterize time-lapse images containing thousands of nascent aggregates [35]. The study of bubble swarm distributions in direct-contact heat exchanger was addressed experimentally [15]. Hence, the knowledge of the shape of the gas region in the gas-liquid two-phase flow is very important to improve the knowledge of the flow structure and flow pattern transition. However, none of the existing studies can provide this information. Aim of this work is that of providing a straightforward procedure able to extract the shape feature parameters of bubbles in a direct-contact evaporator. Also, the details of the hardware configuration and the software and the computations are described in this article. Bubbles images acquisition was carried out as previously referred [14. These images are then treated, analyzed in the following sections.

Out of the above considerations, the remainder of this article is organized as follows. 
Section 2 begins with a direct-contact heat transfer platform manufactured previously 14 and the image processing routines to visualize the bubble formation as well as the medium gas bubble behavior within the direct contact heat exchanger (DCHE), and then introduces some region properties of bubble population, including perimeter, area, equivalent diameter and orientation. Section 3 provides the existing methods for quantifying bubble mixing state and some notable advantages of our investigation. Then above curves and association between a new index for characterizing the flow pattern and the average volumetric heat transfer coefficient, are presented in Section 4 while the conclusion is briefly summarized in Section 5 .

\section{Data acquisition and processing}

\subsection{Experimental study}

The methodology proposed in this study is verified with the feature measurement of direct-contact heat transfer processes in a dynamic system. The experimental set-up used for conducting the evaporation experiments is represented in Fig.1. In all runs, the gauge pressure in the line was kept equal to $1.0 \times 10^{5} \mathrm{~Pa}$. Once the flow rate is determined, the cold fluid is taken to the heating system, which consists of an electric heater whose design temperature ranges from $-160^{\circ} \mathrm{C}$ to $225^{\circ} \mathrm{C}$. Two K-type thermocouples register the operating temperature of the heater. Aiming at minimising the energy loss from the fluid to the surroundings, cotton was coated around this part of the pipeline. The temperature of cold fluid is measured both at the outlet of electric heater and in the chamber on the sparger using K-type thermocouples, whose sensors are located in the middle of the sparger. The evaporator consists of a column $41.3 \mathrm{~cm}$ in inner diameter and $200 \mathrm{~cm}$ in height, at the bottom of which the nozzle, inlet of cold fluid and outlet of thermal fluid are placed and whose top are connected with outlet of cold fluid and inlet of thermal cold. Four thermometers, $50 \mathrm{~cm}$ in length, are placed vertically and isometrically in this column for measuring the mixture temperature.

In this present study, the mixing state of the two-phase flow was analyzed in the DCHE using the fast video recording technique. Bubble spots were filmed with $1280 \times 720$ pixels and RGB Color levels using a high-speed video camera, whose brand is PRAKTICA from Germany and by whose flash in house the required illumination was provided through adjusting the shutter speed setting. To proceed, all the pictures were extracted from the video and analyzed individually. The mixing and morphology of the bubbles were observed by the photographs taken as .bmp files. The following image processing was 
performed with Matlab using the image processing toolbox and in-house functions (i.e., imtophat, im2bw, imfilter, and imopen).

\subsection{Bubbles image preprocessing}

Images were reduced to a window but avoiding the walls of the vessel and other parts of the mixer or devices which could interfere with the analysis. Typical bubbles images from the real DCHT (direct contact heat transfer) process contain a huge amount of bubbles and have a low image quality because of harsh conditions. Once the images extracted and the correct area selected the digital image processing steps were performed. Fig. 2a shows four bubble images of the heat transfer fluids. Digital image processing techniques have been widely used in many process industries for pattern recognition [36], such as the production line of porous powder metallurgy [37]. In this study, the shape feature parameters of bubbles in a direct-contact evaporator are examined by a computer vision technique. The time-lapse images were processed with Matlab using the Image Processing Toolbox (IPT) and in-house functions. The bubble images are also processed by an image processing tool, for instance, ImageJ or more sophisticated methods recently developed. For this current research, we focused on the properties of transient bubbles in DCHE. The visualization of the flow pattern was obtained with the technique described in the previous paragraph. The key steps involved separating each time-lapse image into individual bubbles and surrounding spaces using digital image processing. The sequence of image processing techniques for this purpose is shown in the flow diagram of Fig. 2

As shown in Fig,2, the pictures on the top are the original images and the other three on the bottom are the images after processing. To eliminate noise and enhance the original image (Fig 2 a), the top-hat transform and filter transformation are used here. The implementation of those operations was carried out by means of the in-house functions in IPT, imtophat and imfilter. Segmentation is based on the idea that bubbles are whiter in intensity than the surrounding region in the gray-scale images (Fig.2b). Hence, it is convenient and rational to apply a label for each pixel: pixel within bubbles are labeled one, and the remaining pixels are labeled zero. The gray-scale images obtained by ImageJ are thereafter converted into black-white images to obtain a binary images by applying an appropriated grey level threshold and in-house function im2bw, with white bubbles surrounded by the black spacing (Fig,2 ). More narrowly, im2bw converts the grayscale image to a binary image based on global threshold. Using Otsu's method, the global threshold can be computed by the MATLAB function graythresh and the threshold is chosen to minimize the intraclass variance of the black and white pixels. The initial 
binarization contains multiple bubbles that are very small as well as bubbles that are connected by narrow segments. The open operation implemented by the in-house function imopen is used here to solve this problem. Next, the binary images are converted into the contour images, representing the interface of continuous and dispersed phases (Fig, $2 \mathrm{~d})$. It is shown that the final four contour images corresponding to the images of each column, where the interface between continuous and dispersed phases is shown as black contour lines on a white background.

\subsection{Region properties of bubble population}

In this part, four region properties (perimeter, area, equivalent diameter and orientation) of mixing transient were considered. Ordinarily, the equivalent diameter $D_{\text {eq }}$ is defined as follows,

$$
D_{e q}=\sqrt{\frac{4 A_{e q}}{\pi}}=2 \sqrt{\frac{A_{e q}}{\pi}}
$$
operating conditions. Fig. 3d shows the reported shape characteristic of the bubbles for each transient image. As can be seen from this line graph, the two bubble patterns behave similar in terms of the oscillation of bubble shape apparently. 


\section{Methods for quantifying bubble mixing state}

\subsection{A classical approach for bubbles cluster}

\subsubsection{Ripley's $K$ function and its first application}

Ripley's $K$ function is a widely used tool in spatial point pattern analysis. In order to estimate $K(r)$ from a given bubble pattern on a bounded region $B \subset \mathbb{R}^{2}$, one would obtain the mean number of other points within a distance around every point in $B$ and divide by the estimated intensity. Given an image containing bubbles, $K(r)$ can be obtained as follows. First, the number of bubbles in the recorded image the area of which is denoted by $A$ is counted and denoted by $N$. The intensity $\lambda$ can then be estimated by $\frac{N}{A}$. Let $d_{i j}$ be the Euclidean distance between $i$ th and $j$ th bubbles, which belong to the bubble set in an image, $\{1,2, \cdots, N\}$. Then, the expected point number can be estimated by the average number of points within a given radius $r$ of an arbitrarily chosen point. As such, the estimate of Ripley's $K$ function is:

$$
K(r)=\frac{A}{N^{2}} \sum_{i=1}^{N} \sum_{j \neq i} I\left(d_{i j}<r\right)
$$

where $I\left(d_{i j}<r\right)$ is the indicator function, namely $I\left(d_{i j}<r\right)=1$ when $d_{i j}<r$ is deemed true or $I\left(d_{i j}<r\right)=0$ otherwise. Generally speaking, a larger $K(r)$ implies clustering. Simply, the greater the $K(r)$ value, the severer the clustering.

In this section, we conduct a quantification study of the mixing states using bubble images of two-phases flow samples. Experimental data of two-phase flow parameters were mainly extracted from the flow images. For each image, we compute Ripley's $K$ curve for the first time. MATLAB is used to implement the $K$ function. For these real images, we set $r_{0}=300$ which is the longest distance with which the $K(r)$ is evaluated. Fig. 4 a presents the four curves (for the four bubble images) for Ripley's $K$ function. Observing the curves in this figure, we notice that the 100-sec curve is the lowest line because there is giant bubble agglomerates in the middle with a couple of other bubble blocks scattered in the periphery.

\subsubsection{Classification of total bubbles}

As shown in Fig. 2, the real bubble pattern consists of individual and overlapping or conglutinative bubbles. Despite the desire to break down bubble agglomerates into small pieces of similar sizes, the agglomerates are different to avoid in reality, as evident in the lowest images in Fig. 2, In the case of bubbles classification in a digital image for the parameter estimation, a popular technique is to measure the perimeter and the area of 
set of pixels representing the object and to compute the circularity of an object and it is calculated as follows,

$$
c=\frac{4 \pi A_{r}}{P_{e}^{2}}
$$

where $A_{r}$ is the area and $s$ is the diameter of the elliptical bubble. Fig. 5 shows the independent bubbles (see the upper images in Fig. 5) and overlapping bubbles (see the lower images in Fig. 5) from the lowest images in Fig. 2. According to this figure, it can be seen that the discrete phase accounts for above $50 \%$ of total area, most of which comes from conglutinative bubbles. The resulting flow field inevitably contains bubble agglomerates of various sizes and of irregular shapes. Obviously some mixing or transient processes present better disperse effect, and it is indeed of great interest to the thermal scientists to find out which process or process setting does the job the best.

Overlapping bubbles occur in our experimental cases where quantitative analysis of individual bubbles by their size and shape is desired. Several approaches have been attempted to address the classification and segmentation of the different kinds of bubbles, including the individual bubbles and conglutinative bubbles. Once segmented contours are available, the next step is to deal with overlapping bubbles in a digital image. The real data set consists of images with small and large circular or ellipse-shape bubbles that are randomly distributed. Fig. 6 presents the $K$ curves resulting from single images from Fig. 5. In the panels of this figure, the $K$ curves, corresponding to independent and conglutinative bubbles patterns, in each one are differentiated by using two different line types. It is obviously observed that the plots of these pairwise $K$ curves shed lights in terms of how significantly two types of images are different from each other. Based on the plots, it is apparent that the images of $200 \mathrm{sec}$ are noticeably different from the other three mixing transients. To save space, we omit the presentation of pairwise $K$ curves involving the images at other seconds.

\subsection{Existing uniformity measures for bubbles}

\subsubsection{Topological and statistical measures for validation}

In algebraic topology, the zeroth Betti number $\beta_{0}$ equals the number of connected components that make up the space and the first Betti number $\beta_{1}$ provides a measure of the number of tunnels in the structure [38. The Betti numbers are commonly used to characterize the homogeneity and inhomogeneity of multiphase mixing [3]. Using the image analysis method based on Betti numbers [3], Fig. 7 represents the evolutions of the topological index (Betti numbers) during the mixing. As shown in this figure, the growth of $\beta_{1}$ is superior to $\beta_{0}$ in both growth rate and volatility. The two Betti numbers 
obtained from the cropped image give a better representation of mixing state evolution as compared to that obtained by the raw video images. It is interesting to note that the variation of $\beta_{0}$ and $\beta_{1}$ exhibits fluctuations, which, as expected, are similar to those of the Betti numbers represented in the previous work [3], but those of the $\beta_{1}$ are greater in amplitude, and therefore $\beta_{1}$ represent a more meaningful parameter to study the mixing transient. The picture of the mixture corresponding to representative mixing times are shown. On the basis of the statistical discrepancy method [15], the bubbles uniformity and mixing efficiency in the direct contact heat exchanger have been depicted. However, it is immediately clear that the existing method is influenced by the orientation of the coordinate axes. This limitation will inhibit its use in this application area.

\subsubsection{Notable advantages of our investigation}

In reality, the refrigerating fluid, once injected into the conduction oil, form clusters or agglomerates (bubble swarms) of various sizes and shapes. The existing investigations account the shape features of bubbles within the DCHE. Second, the inner workings of mixing state and its connection with the volumetric heat transfer coefficient can be drawn below (Section 4.2).

\section{Results and discussion} apparently have an influence on bubbles distribution when gas holdup is more than a specific value. It is obviously found that the sum of perimeters of the image in last row of Fig. 2 is larger than that in second row of Fig. 5. With respect to the sum of region 
areas, the same situation occurs. The results show that the two novel flow homogeneitycharacterizing parameters are effective in the real bubble images. In general, the critical parameters of the irregular-shaped bubbles also always depend on fluid characteristics and operation conditions.

In order to further fine verify the feasibility of our method, the evolution feature of bubble patterns has been obtained by means of the existing method (see Fig. 7). The symbols $\mathrm{L}_{1}, \mathrm{~L}_{2}$, and $\mathrm{L}_{3}$ denote the different experimental levels, as depicted in Table 2 The experimental conditions (the height of heat transfer fluid $G_{\text {con }}$, the initial heat transfer temperature difference $\triangle T_{\mathrm{ini}}$, the flow rate of the hot fluid $R_{\mathrm{con}}$ and the flow rate of the refrigerant $R_{\text {dis }}$ ) and heat transfer performance details (average of volumetric heat transfer coefficients $A_{\mathrm{vol}}$ ) are listed. A comprehensive analysis of the proposed method accuracy and repeatability is given below. Fig. 8 represents the evolution curves of the new indices (perimeter and region area of unclassified bubbles) during mixing. They display the same trend of evolution for the two-phase flow, which are constant at low level and then drastically increase. It is noticed that the curves representing the shape feature of bubbles swarm (degree of homogeneity) vary at the beginning of the mixing and rapidly becomes stabilized after fluctuations. Indeed, this observed trend is a very common one for gas-liquid two-phase mixtures. It should be noted that even after a long mixing time, the agglomerates of the present mixture still exist. By taking into account all these considerations, it seems that the image analysis method allows an easy detection of the mixing time as indicated likewise by the Betti numbers evolution. This new method seems more powerful in detecting the influent point than the Betti number $\beta_{1}$ fluctuation signal. The significance of the Betti numbers is that they has been used to intuitively measure the number of connected components and the number of holes for the analysis of the nature of flow structure. Conversely, in the Betti numbers fluctuation signal the detection is very impressionable in terms of bubble size and position, resulting in some cases in a very high uncertainty.

\subsection{Assessment of synergy association}

These previous investigations indicate that the purpose of mixing is to obtain a homogeneous mixture, the local mixing and the flow pattern however has significant effects on the properties of the final products [16, 17, 23]. The initial heat transfer temperature difference, the height of $\mathrm{HTF}$ and the flow rates of fluids are assumed to have the main influence on the bubble generation. In our direct-contact heat transfer system, perimeter and area of bubbles are two important shape-feature parameters of 
system performance. The new index displays the same trend of evolution as for the bubble. It also can been seen that both the two feature parameters can present the difference of bubble shape from beginning to end in the experimental case. There is a linear relationship between the flow patterns of a bubble swarm and heat transfer from the viewpoint of mathematical characterization and experimental analysis. To better compare the data relevant to different systems and conditions, a simplified analysis based on region property (i.e. perimeter of bubbles) of flow patterns is adopted below.

To begin with, the macro-mixing is usually characterized by inflection point (namely, mixing time) $T$ which is an important performance indicator for our two-phase flow stirred system. Over the past years, a lot of measurement methods were appeared to investigate mixing time. There is however no universally accepted method for this issue mainly because each method has its own limitations. Recently, the three-sigma $(3 \sigma)$ method relying on image analysis and statistics was developed to estimate the mixing time accurately [38]. In the paper, we adopt this approach to estimate the mixing time accurately. Assume that $P_{e}^{\text {new }}$ is a new index, combined with mixing time $T$ and it is calculated by $P_{e}^{\text {new }}=\overline{P_{e}} \times \frac{1}{T}$, where $\overline{P_{e}}$ is the average of values and $T$ is the mixing time estimated by our $3 \sigma$ method. Traditionally, a linear relation between $P_{e}^{\text {new }}$ and the average of volumetric heat transfer coefficient $\overline{h_{V}}$ seems to be an outcome as investigated previously [3, 19]. Using the least squares fitting method and the nine experimental levels reported in our previously work, the correlation coefficient is 0.94 and the determination coefficient is 0.8836. Furthermore, this is a special case for quantifying bubbles swarm patterns and heat transfer performance.

\section{Concluding remarks}

The purpose of this work is to measure morphological feature parameters of bubble patterns in a direct-contact evaporator by utilizing image analysis technology. The main conclusions of this paper are briefly summarised as follows:

(1) Four region properties (perimeter, area, equivalent diameter and orientation) of mixing transient at given time points were considered. For each bubble image, we compute Ripley's $K$ curve to quantifying the mixing state from the viewpoint of bubble cluster. Influence of bubbles superposition was also investigated based on this pattern analysis technique and bubbles identification between individual and conglutinative.

(2) Two novel flow homogeneity-characterizing parameters for the purpose of multiphase mixing quantification in the direct-contact heat transfer process were proposed. 
Various aspects of the data acquisition and processing procedures were considered.

It was shown that utilization of the features recorded during three experiments could help provide data that are more reliable. The parameters provide information relating to the final mixing time and the development of mixing during the process.

(3) A comparison with other existing measures reported in the literature indicates that the proposed measure in the current study is promising in improving the evaluation mechanism of mixing uniformity and the establishment of the association between flow pattern and heat transfer performance. It was pointed out that, in agreement with the investigations from [3, 15, 19] related to organic Rankine cycle (ORC) direct contact heat transfer process, care has to be exerted when selecting the fully-mixed criteria in an DCHE.

\section{Acknowledgements}

This work was financially supported by the National Natural Science Foundation of China (Nos. 51666006, 51406071, 51706195 and 51361018), Joint Funds of the National Natural Science Foundation of China (No. U1602272), the Scientific and Technological Leading Talent Projects in Yunnan Province (No. 2015HA019), the Academician Workstation of ZHANG Wenhai in Yunnan Province (No. 2015IC005), and Funds for

International (Regional) Cooperation and Exchange of the National Natural Science Foundation of China (No. 5171101910). We also thank very much Prof. Wuqiang Yang (University of Manchester, UK), Prof. Baofeng Feng (The University of Texas Rio Grande Valley, USA), Prof. Zhibing Zhang and his Ph.D candidate Hongzhou Tian (Nanjing University, PR China) for their essential help. The authors would like to thank

the referees for useful comments and discussion.

\section{Compliance with ethical standards}

The authors declare that they have no conflict of interest.

\section{References}

[1] S. M. S. Murshed, K. Vereen, D. Strayer, R. Kumar, An experimental investigation of bubble nucleation of a refrigerant in pressurized boiling flows, Energy 35 (12) (2010) $5143-5150$.

[2] C. P. Ribeiro Jr, P. L. C. Lage, Experimental study on bubble size distributions in a direct-contact evaporator, Brazilian Journal of Chemical Engineering 21 (1) (2004) 69-81. 
[3] J. Huang, J. Xu, X. Sang, H. Wang, H. Wang, Quantifying the synergy of bubble swarm patterns and heat transfer performance using computational homology, International Journal of Heat \& Mass Transfer 75 (4) (2014) 497-503.

[4] A. Busciglio, F. Grisafi, F. Scargiali, A. Brucato, On the measurement of bubble size distribution in gas-liquid contactors via light sheet and image analysis, Chemical Engineering Science 65 (8) (2010) 2558-2568.

[5] A. Busciglio, F. Grisafi, F. Scargiali, A. Brucato, On the measurement of local gas hold-up, interfacial area and bubble size distribution in gas-liquid contactors via light sheet and image analysis: Imaging technique and experimental results, Chemical Engineering Science 102 (102) (2013) 551-566.

[6] H. Wang, Q. Xiao, J. Xu, Direct-contact heat exchanger, in: S. M. S. Murshed, M. M. Lopes (Eds.), Heat Exchangers - Design, Experiment and Simulation, InTech, Rijeka, 2017, Ch. 07. doi:10.5772/66630.

[7] W. H. Zhang, X. Jiang, Y. M. Liu, A method for recognizing overlapping elliptical bubbles in bubble image, Pattern Recognition Letters 33 (12) (2012) 1543-1548.

[8] F. Ahmed, N. Kawahara, E. Tomita, M. Sumida, Characterization of the spray of the DISI multi-hole injector by means of phase Doppler anemometer, Journal of Thermal Science \& Technology 5 (5) (2010) 36-50.

[9] M. Wang, A. Dorward, D. Vlaev, R. Mann, Measurements of gasliquid mixing in a stirred vessel using electrical resistance tomography (ERT), Chemical Engineering Journal 77 (1-2) (2000) 93-98.

[10] M. I. Hossain, T. Chen, Y. Yang, R. Lau, Determination of actual object size distribution from direct imaging, Industrial \& Engineering Chemistry Research 48 (22) (2009) 10136-10146.

[11] X. Lin, Z. Zhao, Iterative technique for third-order differential equation with threepoint nonlinear boundary value conditions, Electronic Journal of Qualitative Theory of Differential Equatio (12) (2016) 1-10.

[12] L. Dong, X. Li, Y. Qian, D. Yu, H. Zhang, Z. Zhang, Y. Ding, Quantifying nanoparticle mixing state to account for both location and size effects, Technometrics A Journal of Statistics for the Physical Chemical \& Engineering Sciences 59 (2017) $391-403$. 
[13] Q. Xiao, J. Pan, Y. Zhai, Z. Lv, J. Xu, H. Wang, Non-uniformity quantification of temperature and concentration fields by statistical measure and image analysis,

口 Applied Thermal Engineeringdoi:https://doi.org/10.1016/j.applthermaleng. 2017.06 .073

[14] Q. Xiao, J. Pan, Z. Lv, J. Xu, H. Wang, Measure of bubble non-uniformity within circular region in a direct-contact heat exchanger, International Journal of Heat \& Mass Transfer 110 (2017) 5942-5952.

[15] Y. Fei, Q. Xiao, J. Xu, J. Pan, S. Wang, H. Wang, J. Huang, A novel approach for measuring bubbles uniformity and mixing efficiency in a direct contact heat exchanger, Energy 93 (2015) 2313-2320.

[16] D. V. Kalaga, A. Yadav, S. Goswami, V. Bhusare, H. J. Pant, S. V. Dalvi, J. B. Joshi, S. Roy, Comparative analysis of liquid hydrodynamics in a co-current flow-through bubble column with densely packed internals via radiotracing and radioactive particle tracking (RPT), Chemical Engineering Science 170 (2017) 332-346.

[17] D. V. Kalaga, H. J. Pant, S. V. Dalvi, J. B. Joshi, S. Roy, Investigation of hydrodynamics in bubble column with internals using radioactive particle tracking (RPT), AIChE Journal 63 (11) (2017) 4881-4894.

[18] B. Muhumthan, E. Masad, A. Assaad, Measurement of uniformity and anisotropy in granular materials, Geotechnical Testing Journal 23 (4) (2000) 423-431.

[19] Q. Xiao, J. Xu, H. Wang, Quantifying the evolution of flow boiling bubbles by statistical testing and image analysis: toward a general model, Scientific Reports 6 (2016) 31548.

[20] C. P. Ribeiro, P. L. C. Lage, Population balance modeling of bubble size distributions in a direct-contact evaporator using a sparger model, Chemical Engineering Science 59 (12) (2004) 2363-2377.

[21] A.-L. Le Coënt, A. Rivoire, S. Briançon, J. Lieto, An original image-processing technique for obtaining the mixing time: The box-counting with erosions method, Powder Technology 152 (1) (2005) 62-71.

[22] M. I. Hossain, Y. Yang, A. Borgna, R. Lau, Depth-of-field model for size measurement using a borescope imaging technique under high object concentrations, Industrial \& Engineering Chemistry Research 50 (9) (2011) 5824-5830. 
[23] F. Hernandez-Alvarado, D. V. Kalaga, D. Turney, S. Banerjee, J. B. Joshi, M. Kawaji, Void fraction, bubble size and interfacial area measurements in co-current downflow bubble column reactor with microbubble dispersion, Chemical Engineering Science 168 (31) (2017) 403-413.

[24] A. Busciglio, F. Grisafi, F. Scargiali, A. Brucato, On the measurement of local gas hold-up and interfacial area in gas-liquid contactors via light sheet and image analysis, Chemical Engineering Science 65 (12) (2010) 3699-3708.

[25] A. Kalbfleisch, K. Siddiqui, The effect of mesh-type bubble breaker on two-phase vertical co-flow, International Journal of Multiphase Flow 94 (2017) 1-16.

[26] S. Kleinbart, F. Hernandez-Alvarado, D. V. Kalaga, D. Turney, J. Pulido, S. Banerjee, M. Kawaji, Effect of surfactant addition on void fraction distributions measured by a wire mesh sensor, in: ASME 2016 Fluids Engineering Division Summer Meeting Collocated with the ASME 2016 Heat Transfer Summer Conference and the ASME 2016 International Conference on Nanochannels, Microchannels, and Minichannels, 2016, p. V01BT33A009.

[27] Q. Xiao, J. Pan, J. Xu, H. Wang, Z. Lv, Hypothesis-testing combined with image analysis to quantify evolution of bubble swarms in a direct-contact boiling heat transfer process, Applied Thermal Engineering 113 (2016) 851-857.

[28] S. Zafari, T. Eerola, J. Sampo, H. Kälviäinen, H. Haario, Segmentation of overlapping elliptical objects in silhouette images, IEEE Transactions on Image Processing A Publication of the IEEE Signal Processing Society 24 (12) (2015) 5942-5952.

[29] M. Laakkonen, P. Moilanen, T. Miettinen, K. Saari, M. Honkanen, P. Saarenrinne, J. Aittamaa, Local bubble size distributions in agitated vessel: Comparison of three experimental techniques, Chemical Engineering Research \& Design 83 (1) (2005) $50-58$.

[30] R. Kapulla, S. Bravo Najera, Operation conditions of a phase Doppler anemometer: droplet size measurements with laser beam power, photomultiplier voltage, signal gain and signal-to-noise ratio as parameters, Measurement Science \& Technology 17 (2006) 221-227.

[31] G. Xiao, T. Zhou, M. Ni, C. Chen, Z. Luo, K. Cen, Study on oscillating flow of moderate kinetic Reynolds numbers using complex velocity model and phase Doppler anemometer, Applied Energy 130 (5) (2014) 830-837. 
[32] M. Barigou, M. Greaves, A capillary suction prove for bubble size measurement, Measurement Science \& Technology 2 (4) (1991) 318-326.

[33] M. Bailey, C. O. Gomez, J. A. Finch, Development and application of an image analysis method for wide bubble size distributions, Minerals Engineering 18 (12) (2005) 1214-1221.

[34] M. Honkanen, H. Eloranta, P. Saarenrinne, Digital imaging measurement of dense multiphase flows in industrial processes, Flow Measurement \& Instrumentation 21 (1) (2010) 25-32.

[35] C. Xie, H. Zhang, L. J. Shimkets, O. A. Igoshin, Statistical image analysis reveals features affecting fates of Myxococcus xanthus developmental aggregates, Proceedings of the National Academy of Sciences of the United States of America 108 (14) (2011) 5915-5920.

[36] K. C. Fan, J. Torng, W. Jywe, R. C. Chou, J. K. Ye, 3-D measurement and evaluation of surface texture produced by scraping process, Measurement 45 (3) (2012) 384-392.

[37] K. C. Fan, S. H. Chen, J. Y. Chen, W. B. Liao, Development of auto defect classification system on porosity powder metallurgy products, Ndt \& E International 43 (6) (2010) 451-460.

[38] J. Xu, Q. Xiao, Y. Fei, S. Wang, J. Huang, Accurate estimation of mixing time in a direct contact boiling heat transfer process using statistical methods, International Communications in Heat \& Mass Transfer 75 (2016) 162-168. 


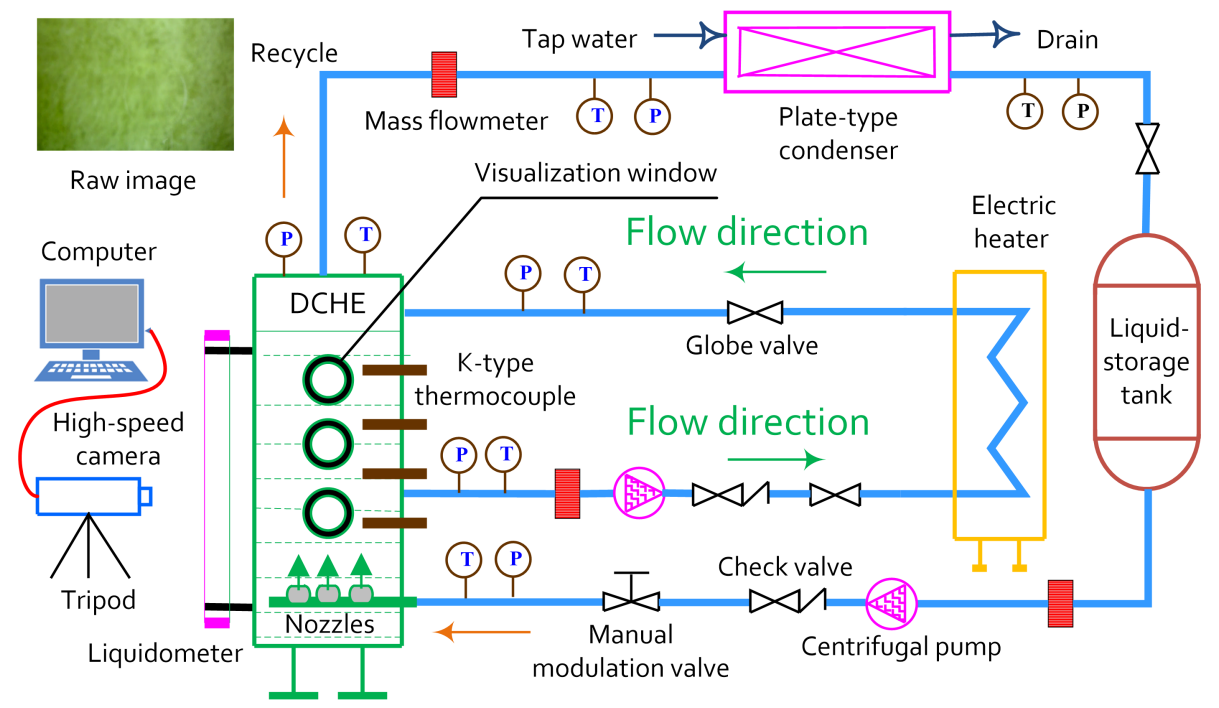

Figure 1: Sketch of the experimental apparatus with optical imaging device for visualization measurements and one raw bubbles image selected randomly. (For interpretation of the references to colour in this figure legend, the reader is referred to the web version of this article.) 


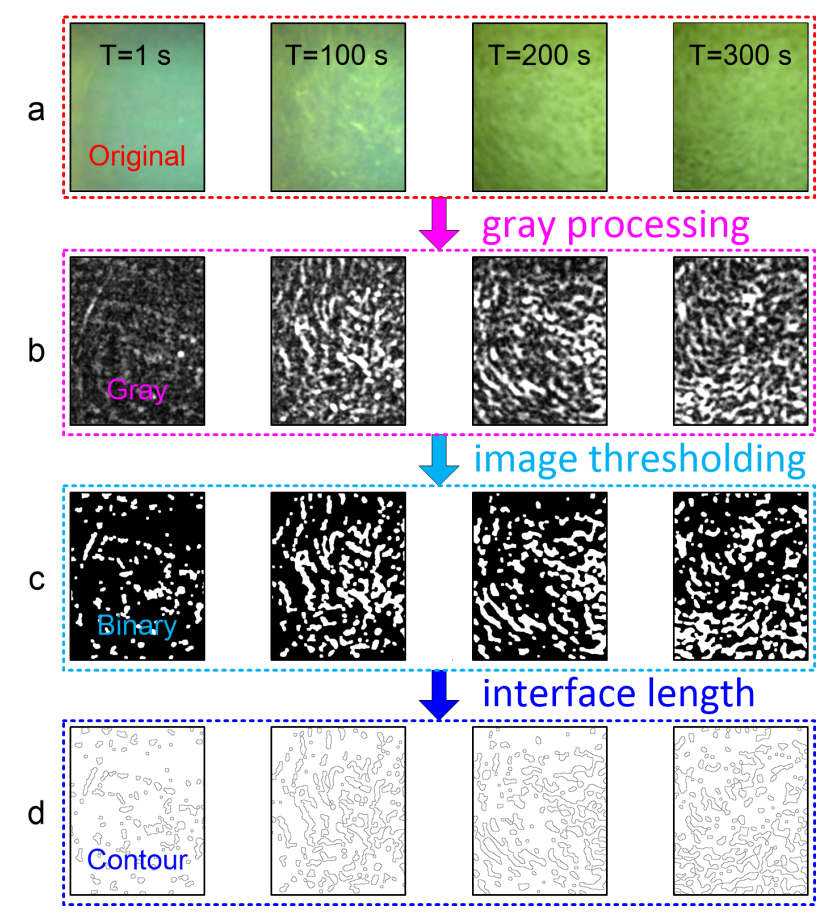

Figure 2: Sequence of operations performed on the series of flow-pattern visualizations taken of sample at each time point: original frames are selected and times are noted in the upper hand part of the frame; lower shows grey-scale, binary and contour images processed in turn for bubble detection. (For interpretation of the references to colour in this figure legend, the reader is referred to the web version of this article.) 

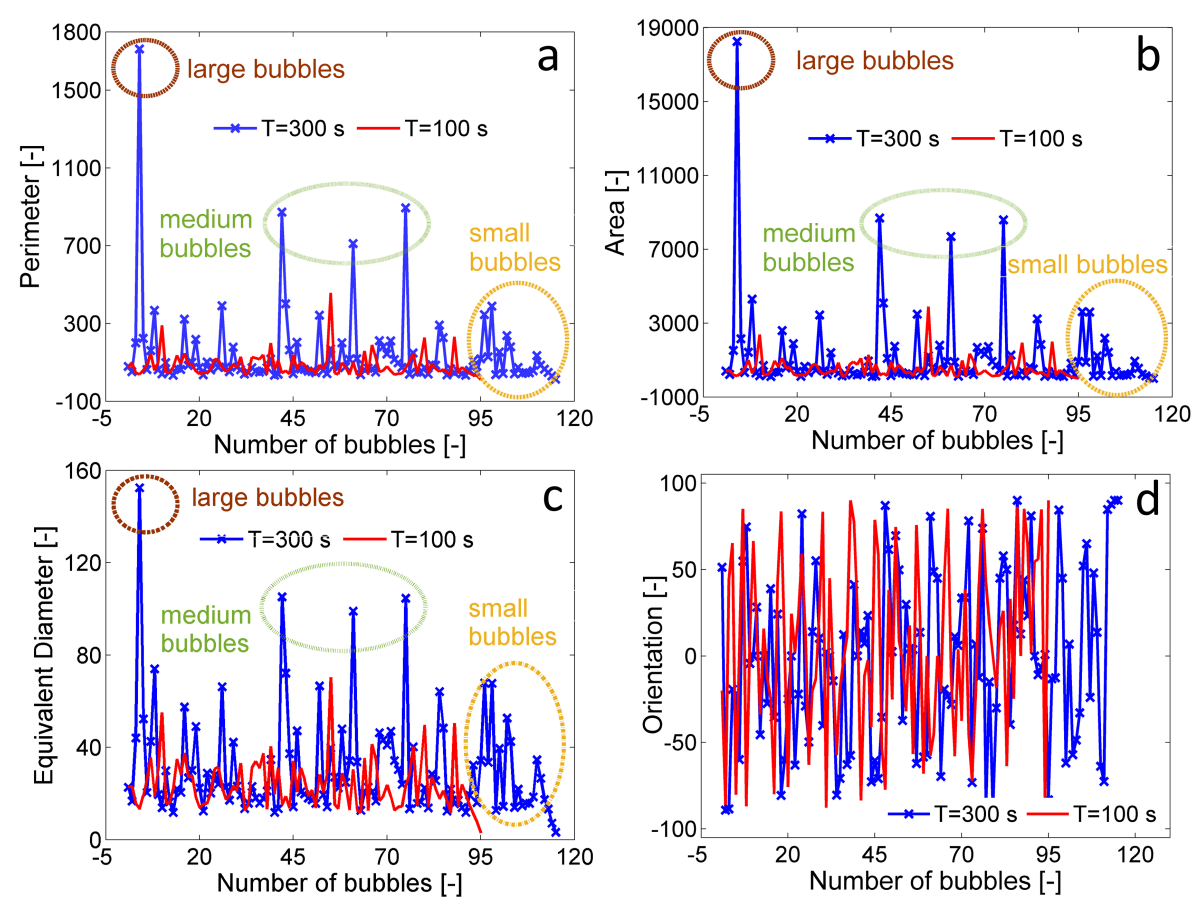

Figure 3: Four region properties of two bubble patterns at two given time points. (For interpretation of the references to colour in this figure legend, the reader is referred to the web version of this article.) 

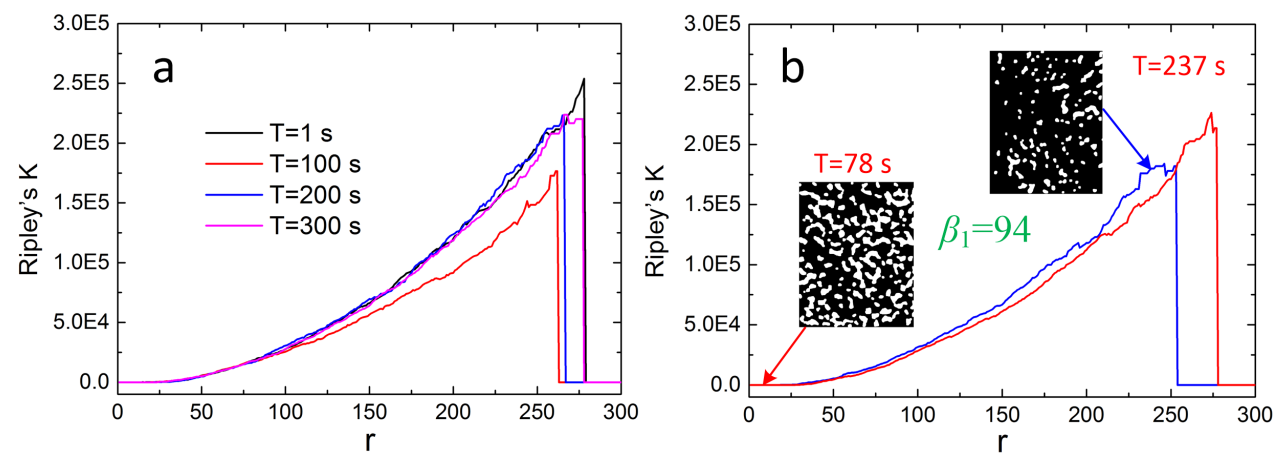

Figure 4: Ripley's $K$ function of bubble processes at four different given time points as shown in Fig. 2 and two special cases $(\mathrm{T}=78 \mathrm{~s}$ and $\mathrm{T}=237 \mathrm{~s}$ ). (For interpretation of the references to colour in this figure legend, the reader is referred to the web version of this article.) 


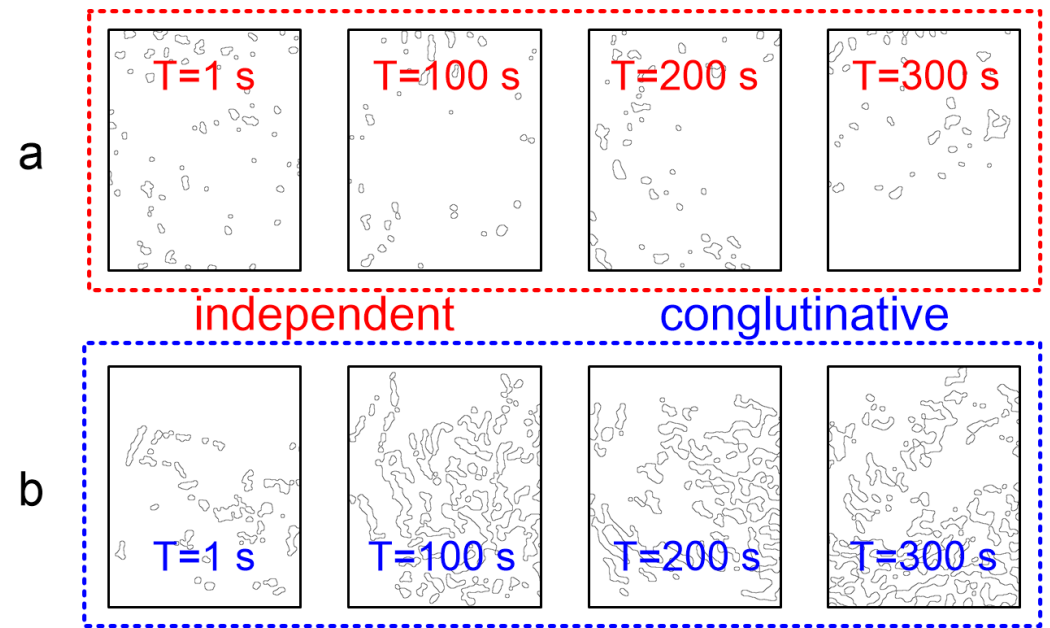

Figure 5: Illustration of independent and conglutinative bubbles by the use of Eq. 3. (For interpretation of the references to colour in this figure legend, the reader is referred to the web version of this article.) 

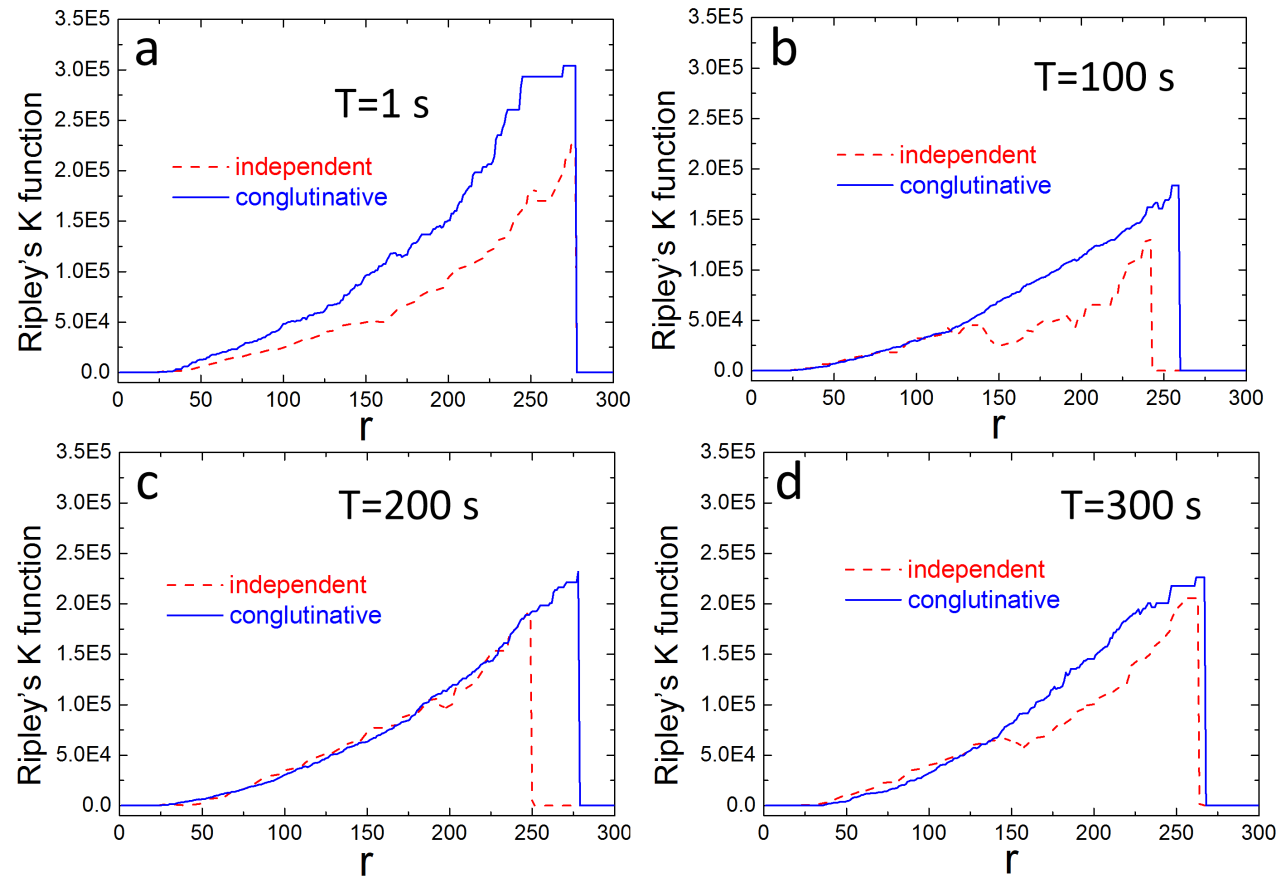

Figure 6: Ripley's $K$ function resulting from single images from Fig. 5 . (For interpretation of the references to colour in this figure legend, the reader is referred to the web version of this article.) 


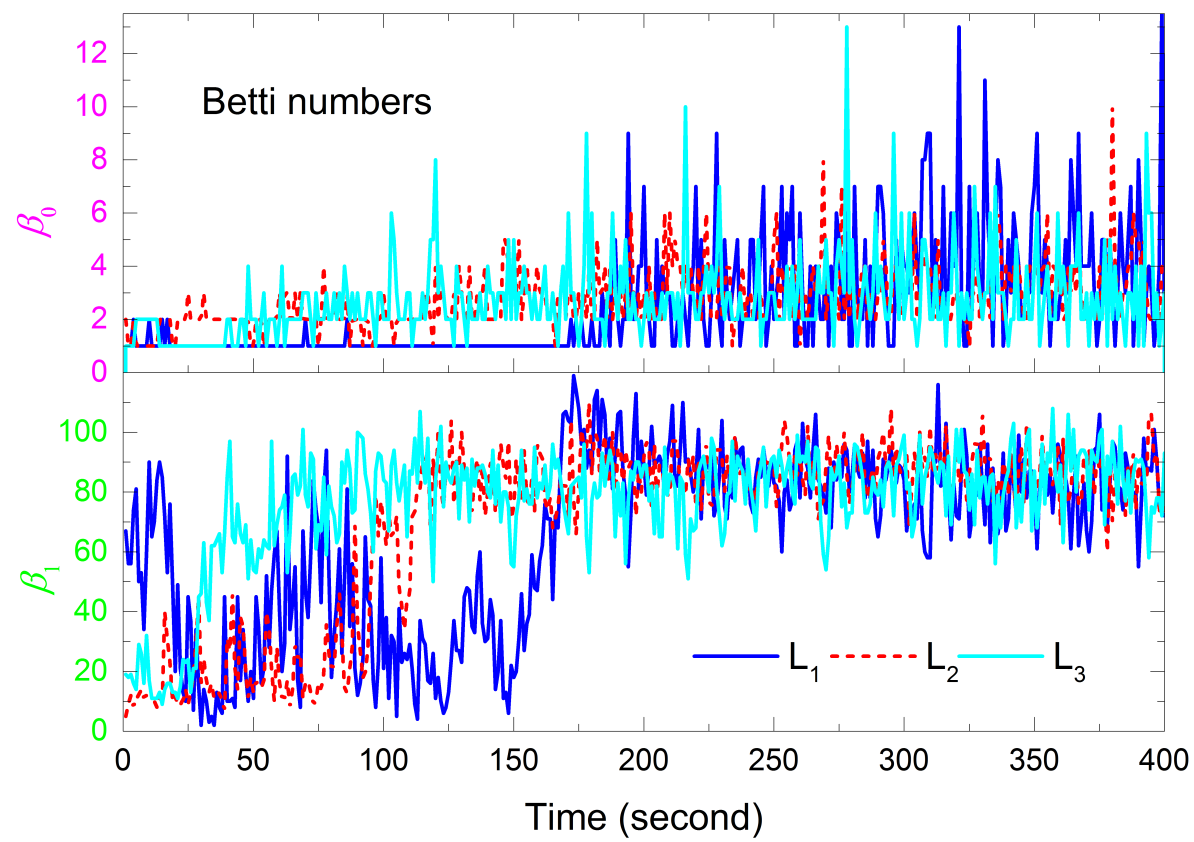

Figure 7: Plots of Betti numbers $\left(\beta_{0}\right.$ and $\beta_{1}$ ) along the mixing process for the three investigated experimental levels $\mathrm{L}_{1}-\mathrm{L}_{3}$. (For interpretation of the references to colour in this figure legend, the reader is referred to the web version of this article.) 


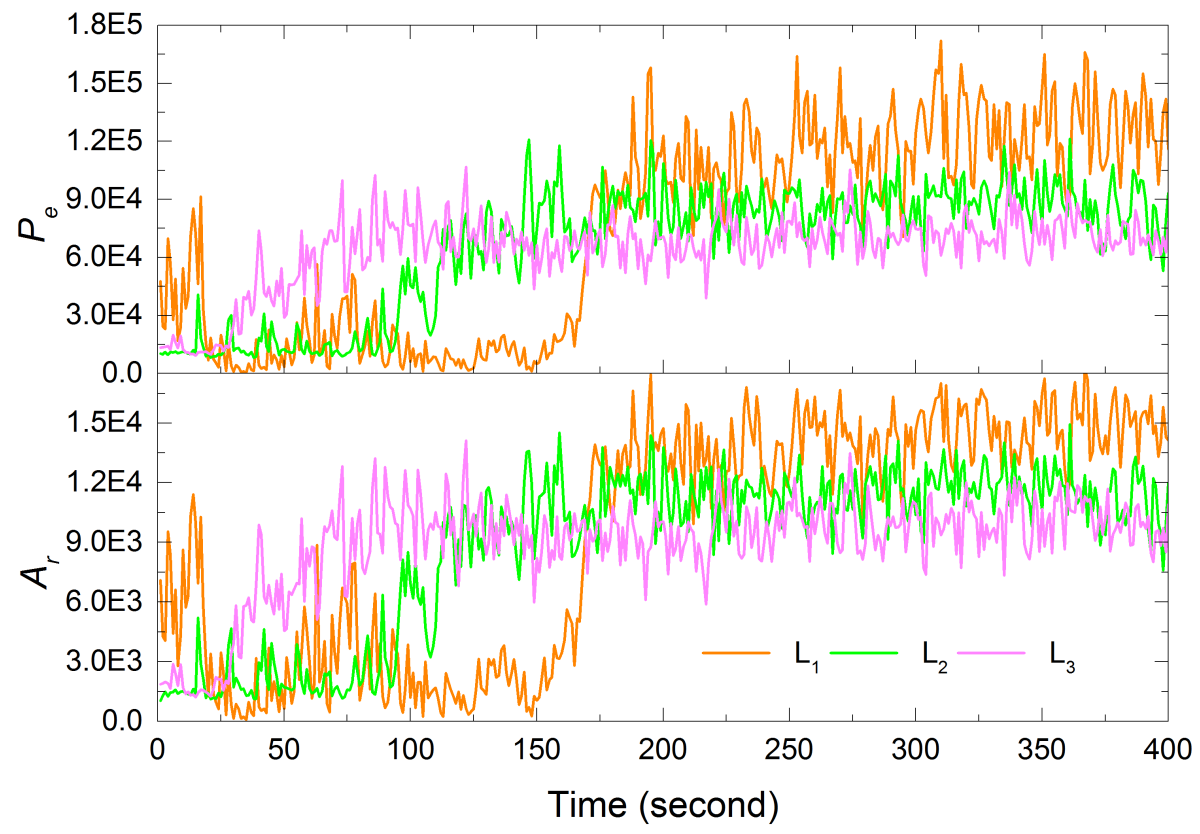

Figure 8: Variation in time (unit: sec) of region perimeters $P_{e}$ and areas $A_{r}$ for three investigated experimental levels $\mathrm{L}_{1}-\mathrm{L}_{3}$. (For interpretation of the references to colour in this figure legend, the reader is referred to the web version of this article.) 
Table 1: Comparison of parameters estimated by digital image processing technology (data unit: pixels).

\begin{tabular}{|c|c|c|c|c|c|}
\hline \multicolumn{2}{|c|}{ Recording time constant } & $\mathrm{T}=1 \mathrm{~s}$ & $\mathrm{~T}=100 \mathrm{~s}$ & $\mathrm{~T}=200 \mathrm{~s}$ & $\mathrm{~T}=300 \mathrm{~s}$ \\
\hline \multirow{3}{*}{$\begin{array}{l}\text { Sum of } \\
\text { perimeters }\end{array}$} & $\begin{array}{l}\text { First row } \\
\text { in Fig. } 5\end{array}$ & $3.9 \times 10^{3}$ & $2.6 \times 10^{3}$ & $3.0 \times 10^{3}$ & $3.4 \times 10^{3}$ \\
\hline & $\begin{array}{c}\text { Second row } \\
\text { in Fig. } 5\end{array}$ & $4.2 \times 10^{3}$ & $1.2 \times 10^{4}$ & $1.3 \times 10^{4}$ & $1.0 \times 10^{4}$ \\
\hline & $\begin{array}{l}\text { Last row } \\
\text { in Fig. } 2\end{array}$ & $8.1 \times 10^{3}$ & $1.5 \times 10^{4}$ & $1.6 \times 10^{4}$ & $1.4 \times 10^{4}$ \\
\hline \multirow{3}{*}{$\begin{array}{l}\text { Sum of } \\
\text { region areas }\end{array}$} & $\begin{array}{l}\text { First row } \\
\text { in Fig. } 5\end{array}$ & 20880 & 14574 & 18693 & 21381 \\
\hline & $\begin{array}{l}\text { Second row } \\
\text { in Fig. } 5\end{array}$ & 28426 & 94125 & 109965 & 92297 \\
\hline & $\begin{array}{l}\text { Last row } \\
\text { in Fig. } 2\end{array}$ & 49952 & 109488 & 129999 & 114078 \\
\hline
\end{tabular}


Table 2: The initial conditions of the three experiments in this study.

\begin{tabular}{ccccc}
\hline Symbol & $G_{\text {con }}$ & $\triangle T_{\text {ini }}$ & $R_{\text {con }}$ & $R_{\text {dis }}$ \\
\hline $\mathrm{L}_{1}$ & $600 \mathrm{~mm}$ & $80 \mathrm{~K}$ & $0.15 \mathrm{~kg} / \mathrm{s}$ & $3 \times 10^{-4} \mathrm{~m}^{3} / \mathrm{s}$ \\
$\mathrm{L}_{2}$ & $530 \mathrm{~mm}$ & $100 \mathrm{~K}$ & $0 \mathrm{~kg} / \mathrm{s}$ & $3 \times 10^{-4} \mathrm{~m}^{3} / \mathrm{s}$ \\
$\mathrm{L}_{3}$ & $530 \mathrm{~mm}$ & $120 \mathrm{~K}$ & $0.15 \mathrm{~kg} / \mathrm{s}$ & $1 \times 10^{-4} \mathrm{~m}^{3} / \mathrm{s}$ \\
\hline
\end{tabular}

